Supporting Information for

\title{
Photoluminescent Quantum Interference in a van der Waals Magnet Preserved by Symmetry Breaking
}

Pingfan $\mathrm{Gu}^{1, \dagger}$, Qinghai Tan ${ }^{2, \dagger}$, Yi Wan ${ }^{1, \dagger}$, Ziling $\mathrm{Li}^{1}$, Yuxuan Peng ${ }^{1}$, Jiawei Lai ${ }^{3}$, Junchao $\mathrm{Ma}^{3}$, Xiaohan Yao ${ }^{1}$, Shiqi Yang ${ }^{1,5}$, Kai Yuan ${ }^{1}$, Dong Sun ${ }^{3,4}$, Bo Peng ${ }^{6}$, Jun $\mathrm{Zhang}^{2,7}$ and $\mathrm{Yu} \mathrm{Ye} \mathrm{e}^{1,4^{*}}$

${ }^{1}$ State Key Laboratory for Artificial Microstructure \& Mesoscopic Physics, Nanooptoelectronics Frontier Center of the Ministry of Education, School of Physics, Peking University, Beijing 100871, China.

${ }^{2}$ State Key Laboratory of Superlattices and Microstructures, Institute of Semiconductors \& Center of Materials Science and Optoelectronics Engineering, University of Chinese Academy of Sciences, Beijing 100049, China.

${ }^{3}$ International Center of Quantum Matter, Beijing 100871, China.

${ }^{4}$ Collaborative Innovation Center of Quantum Matter, Beijing 100871, China.

${ }^{5}$ Academy for Advanced Interdisciplinary Studies, Peking University, Beijing 100871, China.

${ }^{6}$ National Engineering Research Center of Electromagnetic Radiation Control Materials and State Key Laboratory of Electronic Thin Films and Integrated Devices, School of Microelectronics and Solid State Electronics, University of Electronic Science and Technology of China, Chengdu 610054, China.

${ }^{7}$ Beiing Academy of Quantum Information Science, Beijing 100193, China.

$\dagger$ These authors contributed equally to this work. 
Figure S1. Temperature dependent Raman spectra of a layered $\mathrm{CrPS}_{4}$.

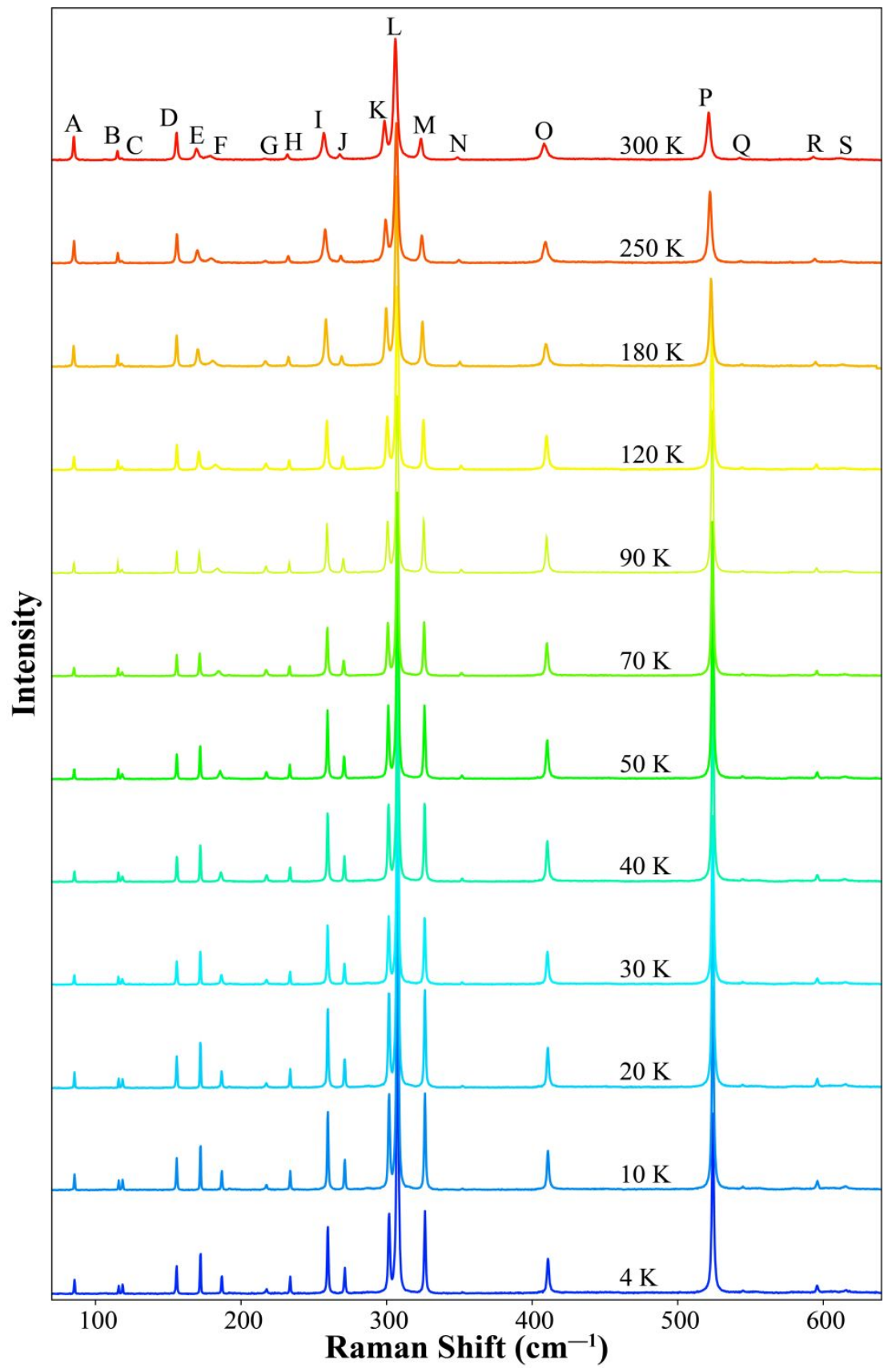

Figure S1. Temperature dependence of Raman spectra of a layered CrPS $_{4} \cdot 19$

Raman peaks are identified at the excitation wavelength of $633 \mathrm{~nm}$ in the range of 70$640 \mathrm{~cm}^{-1}$. The peaks are alphabetically labeled in the order of increasing frequency, and the one at $520.7 \mathrm{~cm}^{-1}(\mathrm{P})$ is resulted from the Si substrate. The frequencies of the peaks at $300 \mathrm{~K}$ and $4 \mathrm{~K}$ are listed in Supporting Materials Table S2. Since the Raman peaks don't show salient changes as the temperature varies, the antiferromagnetic phase transition of $\mathrm{CrPS}_{4}$ is probably uncorrelated with lattice vibrations. 
Figure S2. Angular dependence of the Raman intensities of two peaks under parallel- and cross-configurations
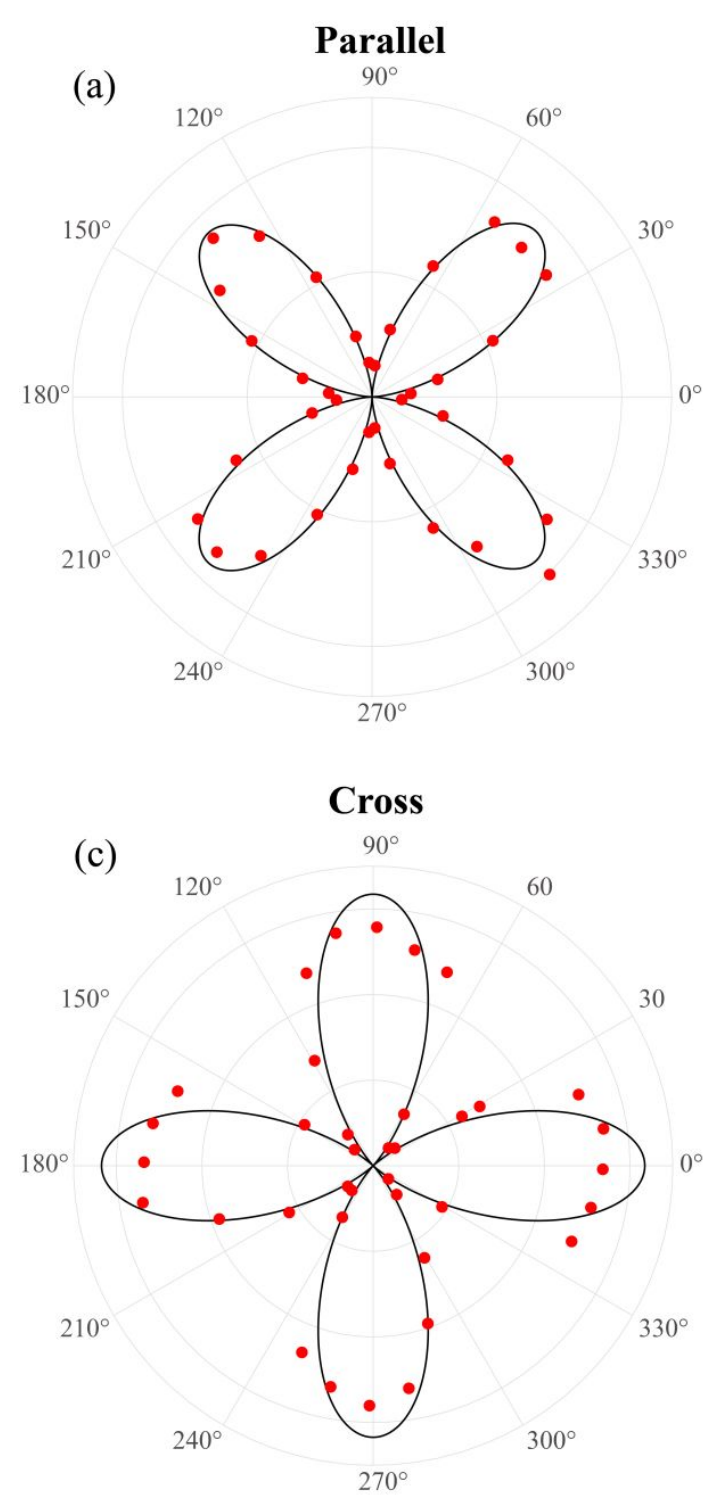

(b)

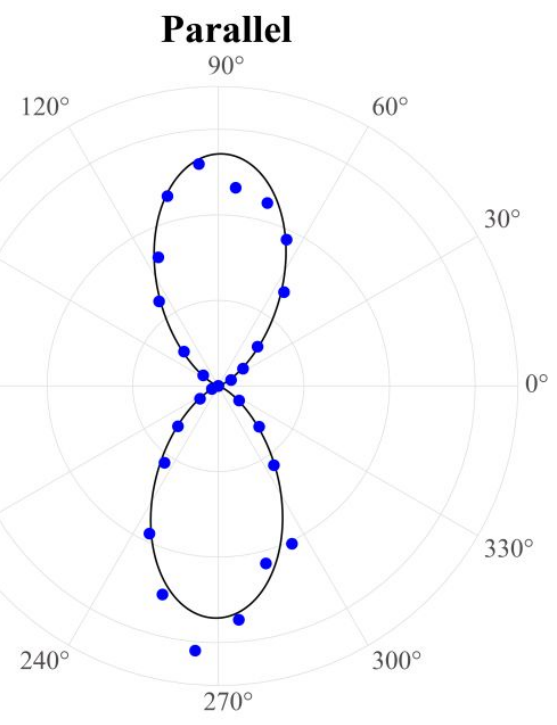

Cross

(d)

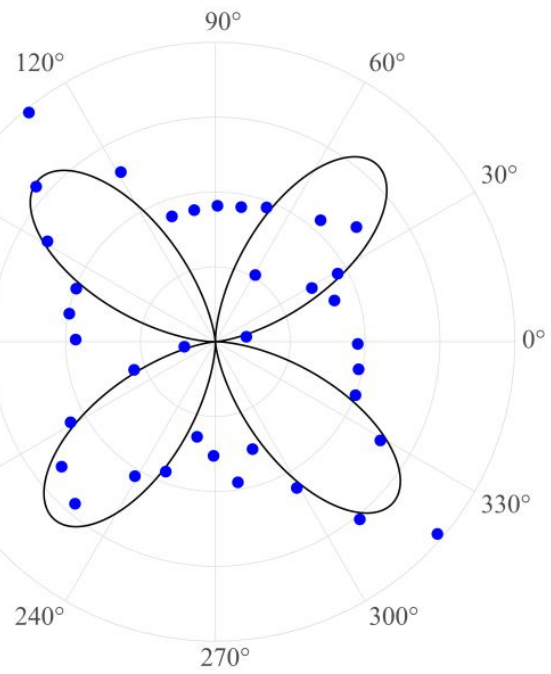

Figure S2. Angular dependence of the Raman intensities of two peaks under parallel- and cross- configurations. The peak at $84.6 \mathrm{~cm}^{-1}$ is assigned to be B mode, showing a four-lobed shape with four maximum intensity angles under both parallelpolarization (a) and cross-polarization (c) configurations. The peak at $169.0 \mathrm{~cm}^{-1}$ is assigned to be A mode, showing a two-lobed shape under parallel-polarization configuration (b) and a four-lobed shape under cross-polarization (d) configuration. 
Figure S3. Polarized PL spectra of layered $\mathrm{CrPS}_{4}$ at room temperature

(a)

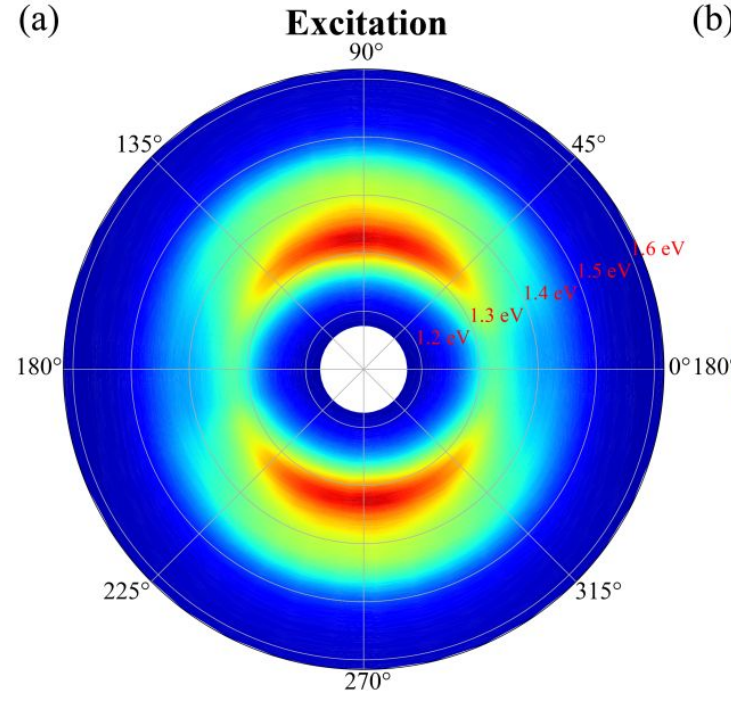

(b)

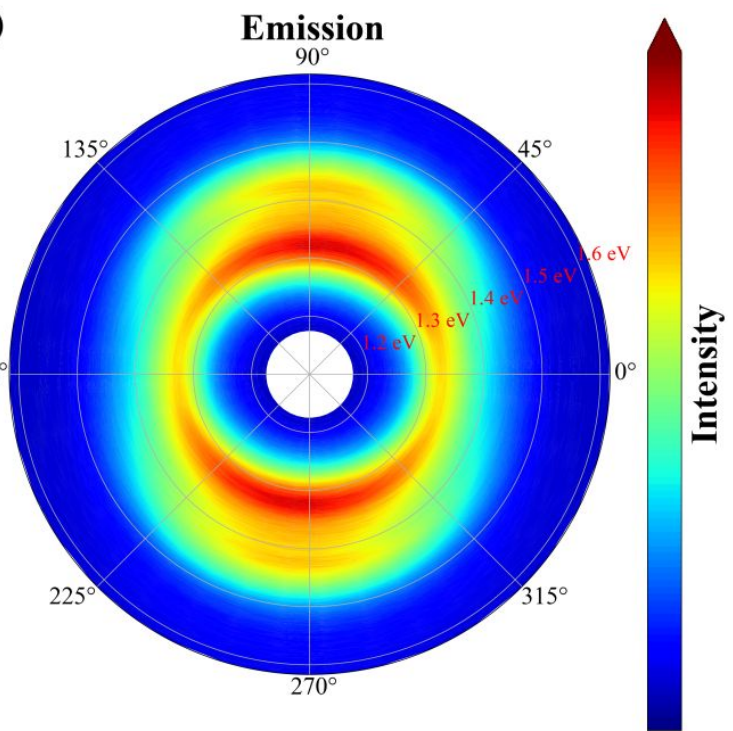

Figure S3. Polarized excitation (a) and emission (b) PL spectra of layered $\mathrm{CrPS}_{4}$ at room temperature. Both the anisotropic patterns can be well-fitted by $\cos ^{2} \theta$ function and are closely aligned with the intrinsic axis of the crystal extracted from the polarized Raman spectra. These features can either be attributed to the anisotropic light-emitting characteristic of single linearly polarized dipole transitions, or to the anisotropic absorption resulted from the in-plane structural anisotropy of the layered $\mathrm{CrPS}_{4}$. 
Figure S4. Normalized PL spectra of $\mathrm{CrPS}_{4}$ on the dependence of excitation laser power at $4 \mathrm{~K}$

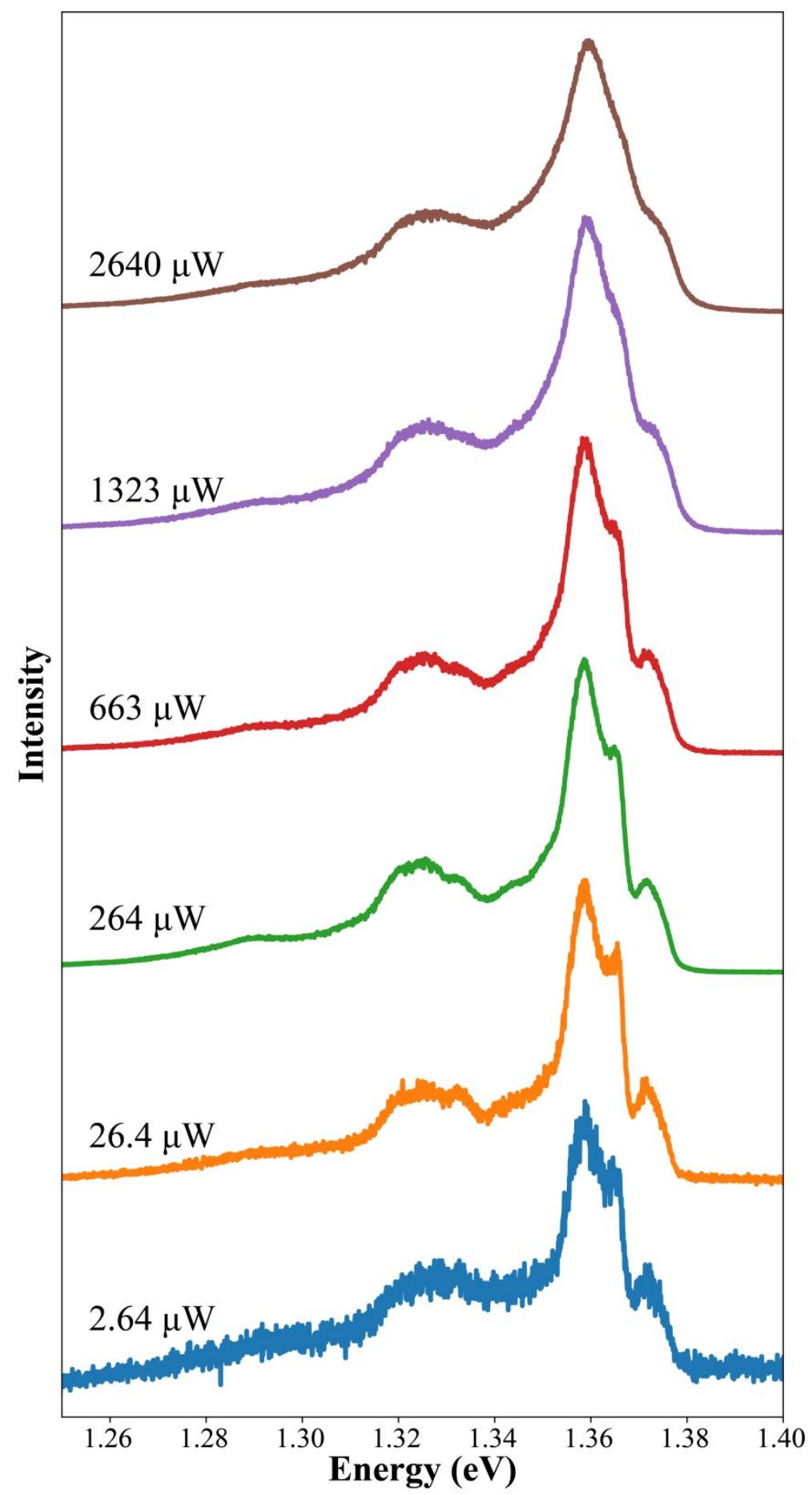

Figure S4. Normalized PL spectra of $\mathrm{CrPS}_{4}$ on the dependence of excitation laser power at $4 \mathrm{~K}$. As the incident power increases, the PL intensity of the continuum states increases linearly while that of the discrete state saturates, leading to the decrease of Fano resonance at high excitation power. 
Figure S5. Polarized PL spectra of layered $\mathrm{CrPS}_{4}$ at $4 \mathrm{~K}$

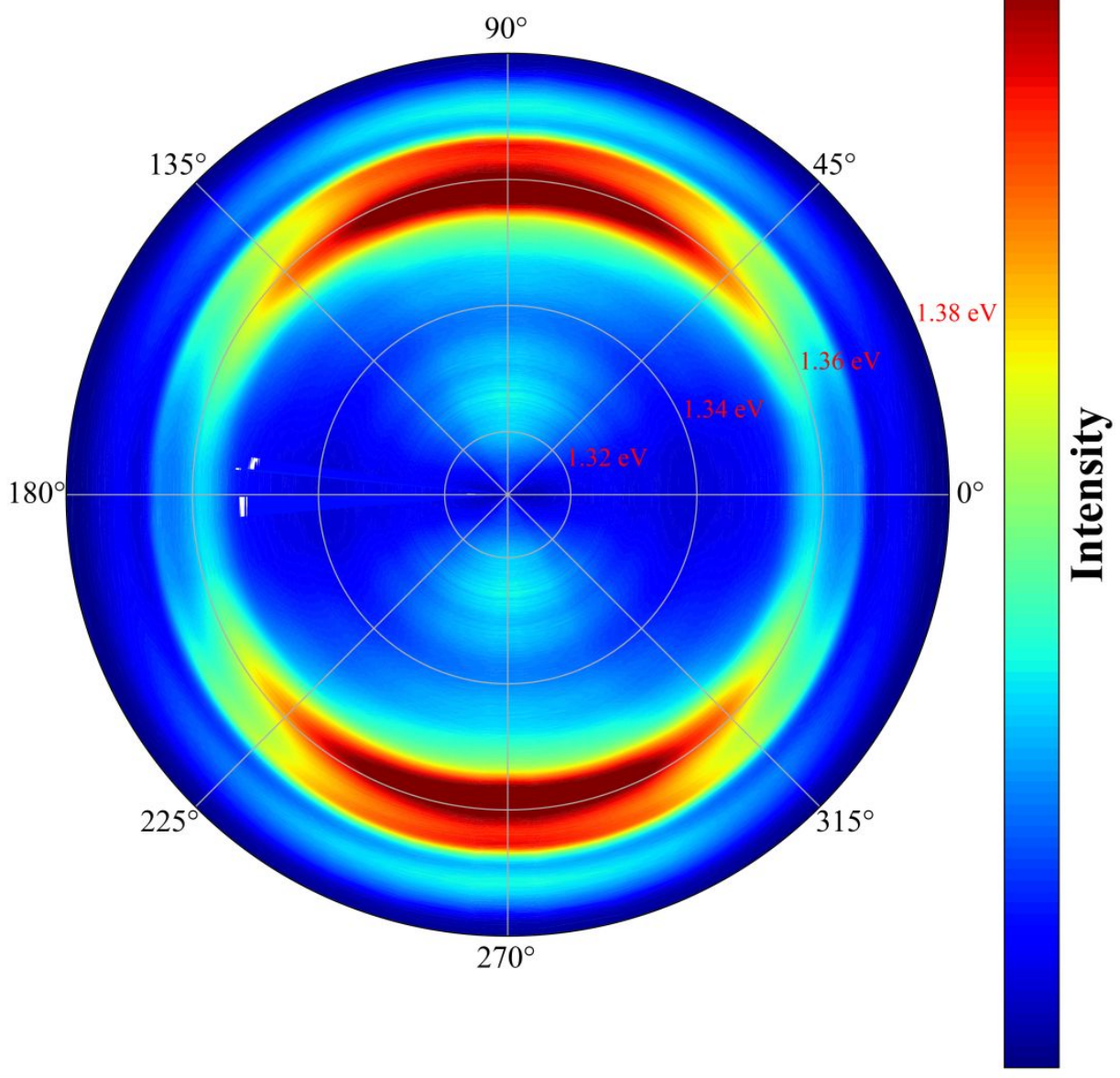

Figure S5. Polarized excitation PL spectra of layered $\mathrm{CrPS}_{4}$ at $4 \mathrm{~K}$. The PL peaks of $\mathrm{CrPS}_{4}$ at $4 \mathrm{~K}$ exhibit identical excitation anisotropy to the peaks at room temperature (Supporting Materials Figure S2), which may indicate that the transitions at low temperature have the same origin as those at room temperature. 
Figure S6. Influence of the $7 \mathrm{~T}$ external field on Fano resonance of $\mathrm{CrPS}_{4}$ at different temperatures.
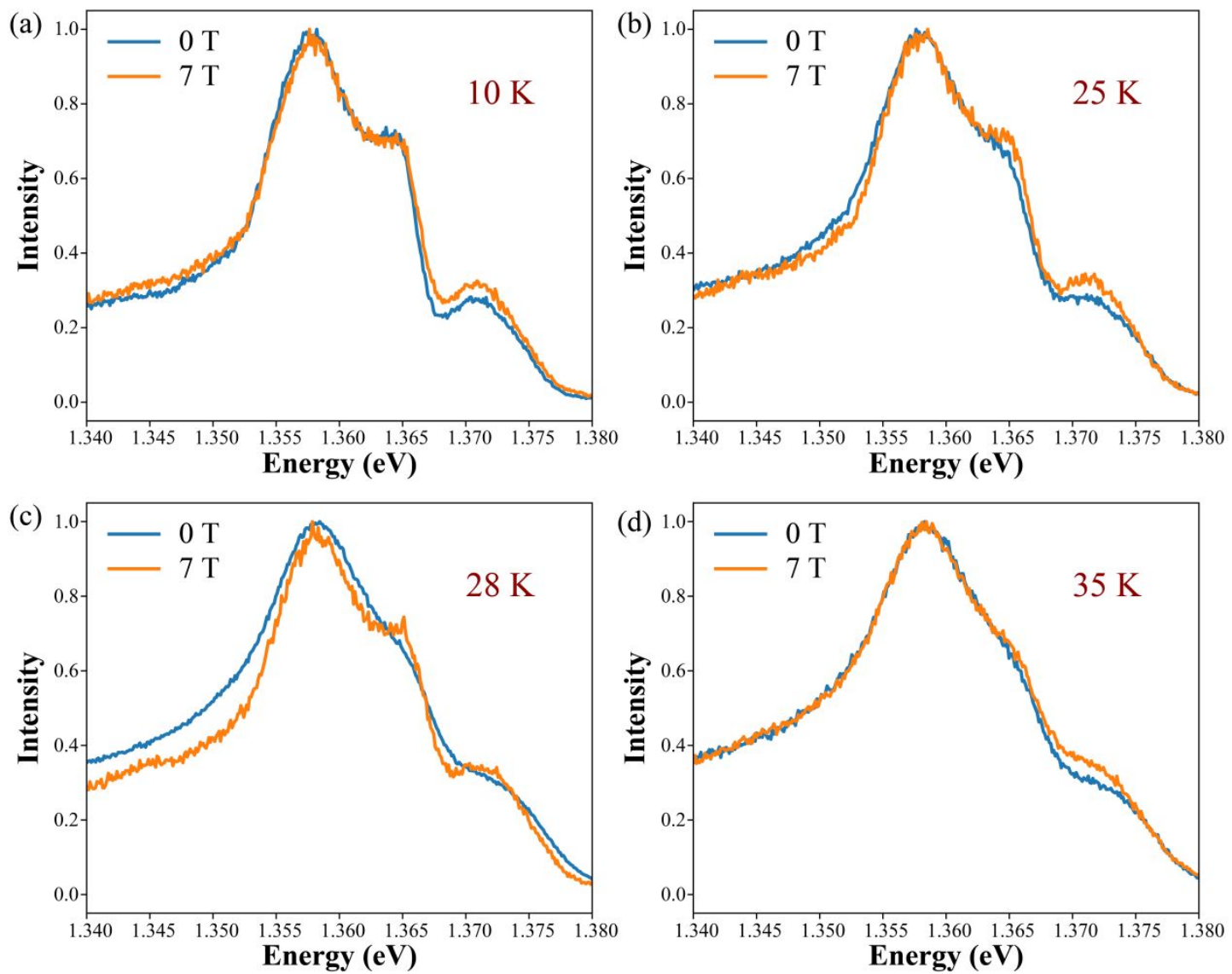

Figure S6. Influence of the $7 \mathrm{~T}$ external field on Fano resonance of $\mathrm{CrPS}_{4}$ at

different temperatures. The Fano resonance is clearly enhanced by the applied field at temperatures between $10 \mathrm{~K}$ and $35 \mathrm{~K}$. At the temperature below $10 \mathrm{~K}$ (above $35 \mathrm{~K}$ ), the applied field shows no obvious influence on the PL spectra. 
Figure S7. Raman spectra of $\mathrm{CrPS}_{4}$ under the applied field.

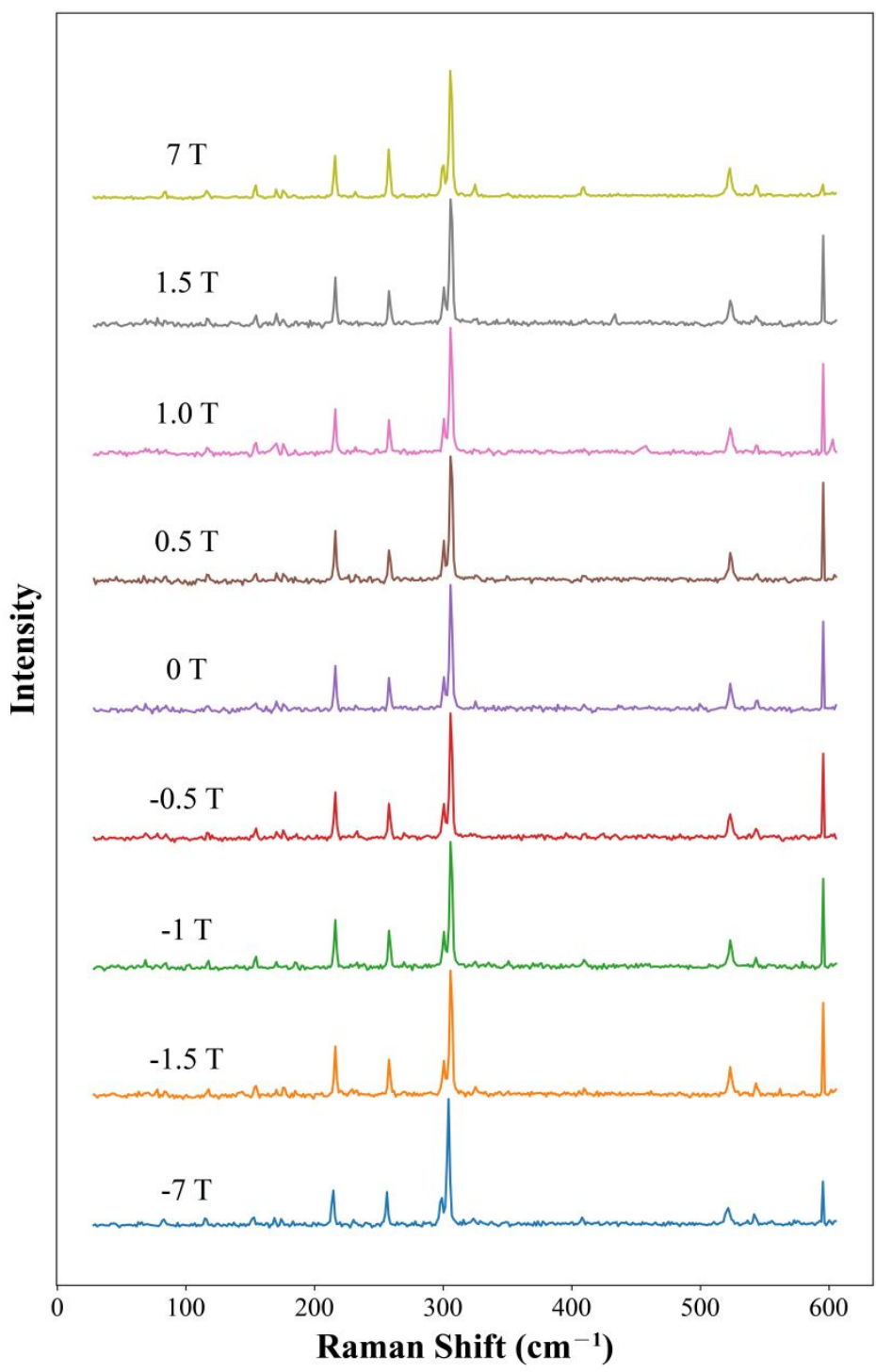

Figure S7. Raman spectra of $\mathrm{CrPS}_{4}$ under the applied field. The Raman spectra of $\mathrm{CrPS}_{4}$ on the dependence of magnetic field exhibit no obvious signature of magnetic behavior, which indicates that the magnetic transition of $\mathrm{CrPS}_{4}$ is unrelated to the lattice vibrations and is consistent with the temperature-dependent Raman spectra. 
Table S1. The chemical composition of layered $\mathrm{CrPS}_{4}$ measured by energy dispersive spectroscopy (EDS)

\begin{tabular}{cccc}
\hline \hline Elements & $\mathrm{Cr}$ & $\mathrm{P}$ & $\mathrm{S}$ \\
\hline Weight\% & 3.67 & 2.67 & 9.00 \\
Weight\% Error & \pm 0.09 & \pm 0.12 & \pm 0.11 \\
Atom\% & 1.80 & 2.19 & 7.14 \\
Atom\% Error & \pm 0.04 & \pm 0.10 & \pm 0.09
\end{tabular}

Table S1. The chemical composition of layered $\mathrm{CrPS}_{4}$ measured by energy

dispersive spectroscopy (EDS). The atomic ratio of $\mathrm{Cr}$ and $\mathrm{S}$ is close to $1: 4$, while the $\mathrm{P}$ is slightly superfluous. 
Table S2. Raman frequencies of the 19 peaks of layered $\mathrm{CrPS}_{4}$ at $300 \mathrm{~K}$ and $4 \mathrm{~K}$.

\begin{tabular}{|c|c|c|c|c|c|}
\hline \multirow{2}{*}{ Peak } & \multicolumn{2}{|c|}{ Frequency $\left(\mathrm{cm}^{-1}\right)$} & \multirow{2}{*}{ Peak } & \multicolumn{2}{|c|}{ Frequency $\left(\mathrm{cm}^{-1}\right)$} \\
\hline & $300 \mathrm{~K}$ & $4 \mathrm{~K}$ & & $300 \mathrm{~K}$ & $4 \mathrm{~K}$ \\
\hline A & 84.6 & 85.1 & $\mathrm{~K}$ & 298.0 & 301.1 \\
\hline B & 114.6 & 115.4 & $\mathrm{~L}$ & 305.5 & 306.9 \\
\hline $\mathrm{C}$ & 117.5 & 118.1 & M & 323.0 & 325.8 \\
\hline $\mathrm{D}$ & 155.1 & 155.2 & $\mathrm{~N}$ & 348.1 & 350.4 \\
\hline $\mathrm{E}$ & 169.0 & 171.6 & $\mathrm{O}$ & 407.9 & 410.4 \\
\hline $\mathrm{F}$ & 177.9 & 186.2 & $\mathrm{P}$ & 520.7 & 523.6 \\
\hline G & 215.7 & 217.0 & Q & 542.1 & 544.1 \\
\hline $\mathrm{H}$ & 231.3 & 233.2 & $\mathrm{R}$ & 592.7 & 595.3 \\
\hline I & 256.4 & 259.0 & S & 609.9 & 614.9 \\
\hline $\mathrm{J}$ & 267.3 & 270.7 & & & \\
\hline
\end{tabular}

Table S2. Raman frequencies of the 19 peaks of layered $\mathrm{CrPS}_{4}$ at $300 \mathrm{~K}$ and $4 \mathrm{~K}$. 